\title{
Metodología de producción para el desarrollo de contenidos audiovisuales y multimedia para MOOC
}

\section{Production methodology for the development of audiovisual and multimedia content for MOOC}

\author{
Manuel Gértrudix Barrio \\ Mario Rajas Fernández \\ Sergio Álvarez García \\ Universidad Rey Juan Carlos (España)
}

\section{Resumen}

El desarrollo de un MOOC de calidad supone la puesta en marcha de un complejo proceso de planificación, diseño y desarrollo que implica a diferentes profesionales y áreas de trabajo. La eficacia del sistema de producción precisa establecer metodologías específicas que atiendan, no solo a las características concretas del contexto de elaboración, sino que aúnen estrategias y técnicas procedentes de diferentes ámbitos como el diseño de recursos educativos, la producción audiovisual y el desarrollo multimedia e interactivo. A través de la aplicación de un proceso inspirado en los principios de investigación-acción, se presenta el modelo metodológico alcanzado para la producción y desarrollo de contenidos audiovisuales y multimedia para MOOC, tras un año de interacciones sucesivas. El proceso se ha llevado a cabo durante el desarrollo secuencial de los cinco primeros MOOC elaborados para la plataforma URJCx de la Universidad Rey Juan Carlos. El proceso ha requerido la configuración de equipos de trabajo y medios especializados, de un programa de acción, y de la definición de estrategias que han sido puestas a prueba mediante procesos iterativos y participados de diseño, implantación (acción), análisis y evaluación dirigidos a la mejora y la eficacia de los procesos de producción. La secuencia de interacciones han permitido definir un modelo de producción más ligero, basado en el desarrollo de esquemas y patrones formales que funcionan como plantillas generales tanto para la producción audiovisual como multimedia, y la mejora de los flujos de comunicación y trabajo entre los equipos de trabajo involucrados.

Palabras clave: MOOC; enseñanza a distancia; educación multimedia; investigación-acción; producción audiovisual.

\begin{abstract}
The development of a MOOC involves the implementation of a complex process of planning, design and development. This process requires the participation of different professionals and work areas. The efficiency of the production system needs to establish specific methodologies. These should address the specific characteristics of the context of development, and they must combine strategies and techniques from different areas:
\end{abstract}


instructional design, audiovisual production and multimedia development. The article presents a methodological model for the production and development of audiovisual and multimedia content for MOOC, after a year of successive interactions of implementation and evaluation. This becomes a result of a procedure based on the action research methodology. The process has been carried out during the sequential development of the first five MOOCs developed for the platform URJCx Rey Juan Carlos University (Spain). The process, which is part of an overall strategy, has required the creation of dedicated professional teams and the provision of specialized resources. The working methodology has been tested by iterative processes of design, implementation (action), analysis and evaluation aimed at improving the efficiency of production processes. The interactions sequence? have defined a model of lighter production, based on the development of schemes and formal patterns both for audiovisual production and multimedia. These schemes work as general templates. Also it has improved communication flows and work among the teams involved.

Keywords: MOOC; distance education; multimedia instruction; action research; audiovisual production.

Es indudable ya que, más allá del debate abierto sobre el impacto real y la influencia de los MOOC (White et al., 2013; García Aretio, 2015), la fase inicial de explosión del fenómeno está dando lugar a una nueva etapa de análisis detallados y reflexiones de mayor amplitud, que tratan de superar el escenario inicial de precipitación en la implantación de iniciativas señalado por Aguaded (2013), y que van despejando algunas de las incógnitas que para las universidades, señaladamente las iberoamericanas, vislumbraban Capdevilla y Aranzadi (2014). El fenómeno está siendo ampliamente tratado en la literatura académica en un recorrido que va desde análisis bibliométricos que miden la representación del concepto en la literatura científica y, por tanto, su interés como objeto de estudio (López, Vázquez y Román, 2015), el examen de su calidad pedagógica (Vila, Andrés y Guerrero, 2014; Aguaded y Medina-Salguero, 2015) o las políticas institucionales que los impulsan (Hollands y Tirthali, 2014a; Haywood y Macleod, 2014), entre otros muchos aspectos. En una era de post-escasez del conocimiento, la plétora anunciada, entre otras corrientes, por los MOOC alumbra nuevos fenómenos de micro-masas basadas en la "agregación de la larga cola, la masa de los pequeños (pero poderosos) colectivos" (Jarvis, 2010, p. 94) y, sin saber cómo cristalizará, parece evidente que nada quedará igual en el marco de este nuevo “auleph” tecnológico (Álvarez, 2010, p. 304).

La puesta en marcha de una nueva propuesta en este ecosistema requiere la definición previa de una estrategia global que no solo se incardine con la misión y visión de la institución y con sus objetivos principales sino que también asuma globalmente los requisitos y necesidades, tanto técnicos como de recursos y procesos de producción necesarios, de forma que se garantice el desarrollo de productos de calidad en un marco de sostenibilidad a medio y largo plazo. Como señalan Hollands y Tirthali: "Given the considerable investment that MOOCs represent, we recommend that prior to embarking on MOOC-related initiatives, institutions carefully consider 
their goals and whether MOOCs present a realistic and financially justifiable means to achieve them" (2014, p. 1).

Este aspecto, el de la sostenibilidad de la acción, resulta esencial, especialmente en un marco económico exigente que requiere maximizar los recursos que se ponen a disposición, y que se encuentra sujeto a las nuevas fuerzas de ese procomún colaborativo de coste marginal cero que enuncia Rifkin también para la educación y que está transformando el paradigma de la enseñanza superior (2014). Encontrar esquemas de producción que se ajusten a modelos de negocio viables y sostenibles resulta esencial para conciliar los objetivos perseguidos con la durabilidad de la puesta a disposición de nuevos cursos, tal como ha sido ya documentado en distintas experiencias como la austriaca (Fischer et al., 2014), la alemana Stifteverband (Lorenz, 2016) o la británica a través de Futurelearn (Sánchez, León y Davis, 2015).

\section{METODOLOGÍA}

El diseño metodológico se fundamenta en una aplicación adaptada de los procesos de investigación-acción (Rodríguez y González, 2013) desde la dimensión tanto de la mejora de los programas educativos de los MOOC como, de forma especial, los sistemas de planificación y desarrollo de esta tipología de cursos. En un contexto de fuerte cambio en las dinámicas educativas a nivel global (GonzálezSancho y Vincent-Lancrin,2016), sometidas a una adaptación de los procesos de aprendizaje a las lógicas líquidas de una sociedad globalizada (Best, 2015), resulta esencial realizar aproximaciones críticas que, a partir de una interacción constante y retroalimentada entre conocimiento y actuación, permitan obtener los mejores resultados en términos prácticos, adaptándose estos a las necesidades reales tanto del contexto en el que se desarrollan como de la comunidad de destino.

Siguiendo la secuencia de las fases y evaluación estándar de la IA definidos por Lewin (como se cita en Elliot, 2005), desde una perspectiva de aplicación concreta al desarrollo de productos similar a la realizada por otras investigaciones (Contreras, Eguia y Solano, 2016), se presenta el modelo metodológico alcanzado para la producción y desarrollo de contenidos audiovisuales y multimedia para MOOC, tras un año de interacciones sucesivas basadas en ciclos recursivos de planificación, acción, observación y reflexión.

Se ha optado por este enfoque metodológico ya que permite abrir la participación a todos los equipos involucrados en el proceso de elaboración de los MOOC, tiene una clara orientación práctica que permite aprender del proceso y de los errores cometidos durante este, y permite evolucionar en espiral en la medida que crece la dimensión del proyecto y su alcance (Given, 2008). El objetivo es hacer una evaluación crítica de los modelos de diseño y producción de este tipo de cursos masivos abiertos en línea con la finalidad de establecer una metodología eficiente para la práctica educativa, en los términos establecidos por Carr y Kemmis (1986). De este modo, el proceso se ha llevado a cabo durante el desarrollo secuencial de los 
cinco primeros MOOC diseñados para la plataforma URJCx, dentro de la estrategia general de contenidos abiertos de la Universidad Rey Juan Carlos.

En el proceso de reconocimiento y revisión previa se han problematizado las fases del proceso de producción a través de un conjunto de preguntas que permitiesen describir los hechos iniciales de la situación considerando los siguientes ámbitos: estratégico, modelización del MOOC, optimización del proceso de producción audiovisual, y optimización del proceso de producción multimedia. A partir de estos, se ha elaborado el plan general de actuación y se han puesto en marcha las sucesivas etapas de acción.

\section{RESULTADOS}

\section{Contexto de producción}

\section{Iniciativa de la Universidad Rey Juan Carlos}

Los MOOC (Massive Open Online Courses) representan un ámbito de disrupción educativa cuyos alcances, en un proceso global de transformación digital de las instituciones de Educación Superior (Balkin y Sonnevend, 2016), resultan de gran interés como objeto de estudio. A pesar del incremento reciente de los trabajos que los examinan, aún resultan insuficientes los estudios empíricos que analicen detenidamente tanto sus modelos como sus efectos (Bartolomé y Steffens, 2015). No es discutible, sin embargo, ni el despliegue de su creciente oferta ni cómo ello está generando un nuevo escenario de formación abierta en el que numerosas instituciones universitarias internacionales están participando de manera incremental -en una estimación de Class-Central para 2015, más de 500 universidades, 4.200 cursos y 35 millones de estudiantes (2015).

Hollands y Tirthali (2014), en una investigación de largo alcance, han identificado seis objetivos principales que motivan en las instituciones la puesta en marcha de iniciativas de desarrollo de MOOC: ampliar el alcance y acceso al conocimiento; contribuir a afianzar la marca; mejorar la productividad reduciendo costes o aumentando los ingresos; mejorar los resultados educativos; producir innovación en la enseñanza y el aprendizaje; o la realización de investigaciones sobre nuevos modelos educativos.

En este contexto, la Universidad Rey Juan Carlos ha establecido una línea de acción dirigida a afianzar y ampliar su estrategia de formación abierta a distancia, sumándose a las iniciativas implementadas con Open edX a través de la puesta en marcha de una plataforma propia y del lanzamiento de una convocatoria dirigida al diseño, desarrollo e impartición de MOOC para dicha plataforma: URJCx.

Los principales objetivos perseguidos son: la apertura de los procesos de formación y conocimiento; la introducción de lógicas de conocimiento abierto en 
el seno de la institución; fraguar dinámicas de cambio y transformación digital; proyectar la marca y el compromiso social de la universidad; proveer contenidos de calidad en áreas de interés, actualidad y alcance internacional; dirigirse a sectores y perfiles laborales con alta demanda de empleo; y alinearse con los retos de la Agenda Digital Europea 2020. A estos objetivos se suma el disponer de una plataforma que facilite el despliegue de otras configuraciones formativas flexibles como SPOC y NOOC (NANO Curso Abierto, Masivo y en Línea) (INTEF, 2016), que permitan atender la demanda formativa de acciones transversales como los módulos de Reconocimiento Académico de Créditos destinados a los más de 5.00o estudiantes de nuevo ingreso que recibe la universidad cada año.

La iniciativa se incardina dentro de un proceso global de extensión de la oferta formativa online de la URJC (Gértrudix, Rajas y Esteban, 2015) que ha sido acompañada con la creación de una unidad específica: el Centro de Innovación en Educación Digital - URJC online (CIED), que da soporte a esta acción. Este aspecto, de configuración de estructuras formales dentro de la institución, resulta fundamental para la optimización de los procesos y los costes, y para fomentar la longevidad de la iniciativa, tal como sucede en otras instituciones internacionales: "A few institutions have created offices of online learning to facilitate the development of MOOCs and other online and blended courses" (Hollands y Tirthali, 2014, p. 134).

\section{Fases del proyecto}

El proyecto se ha llevado a cabo en seis fases: análisis estratégico, análisis de las soluciones tecnológicas, convocatoria de cursos, desarrollo de la plataforma, elaboración de la primera serie de cursos, despliegue de ellos y evaluación de resultados.

1. Análisis estratégico. A partir del análisis de la oportunidad de poner en marcha una iniciativa de MOOC propia de la universidad, a comienzos del año 2014 se comenzó un estudio detallado de las plataformas, modelos de cursos y soluciones existentes y se definió una estrategia global de la institución en el ámbito de la educación abierta, estableciendo como línea estratégica la puesta en marcha de una solución propia para el despliegue de MOOC, SPOC (Small Private Online Course) y formación masiva interna.

2. Análisis de las soluciones tecnológicas. La Universidad Rey Juan Carlos carecía de una plataforma específica de Cursos Masivos Online Abiertos que escalase para miles de usuarios concurrentes en un curso y que se adaptara a los flujos de trabajo que requiere este modelo formativo. Finalmente se optó por Open edX porque, dentro de la diversidad de plataformas existentes, se trataba de la solución abierta más utilizada. A finales del año 2014 se realizó una prospectiva y estudio del mercado internacional de providers de soluciones bajo Open edX (IBL Studios, Appsembler, Accredible, EdCast y Jazkarta), y se optó finalmente 
por una solución que integrase, además del core de Open edX, servidor de badges, modelo de integración con soluciones de Microsoft como Office 365, sistema de monetización mediante pasarelas de pago como PayPal, o gestión integrada con CMS como WP o Joomla.

3. Convocatoria de cursos. En el mes de abril de 2015 se lanzó una convocatoria abierta al profesorado de la Universidad Rey Juan Carlos para proponer la realización de cinco MOOC. El objetivo de la convocatoria era el desarrollo de Cursos Abiertos y Masivos en Línea de calidad que pudiesen ser impartidos por docentes de la universidad dentro de la plataforma URJCx y siguiendo los estándares de calidad establecidos por el consorcio edX. Las propuestas presentadas debían estar orientadas a la producción de MOOC que, por su temática y planteamiento didáctico, pudiesen resultar de interés para un número importante de estudiantes nacionales e internacionales, contribuyendo con ello a proyectar e impulsar la visibilidad de la URJC. Fruto de esta convocatoria a la que se presentaron 18 propuestas, se seleccionaron, por un jurado internacional experto en MOOC, las siguientes cinco: Ciberseguridad. Ataques y contramedidas; Ciencia, Gestión e Ingeniería de Servicios; Emprendimiento. Cómo gestionar el desarrollo tecnológico de una Startup; The Chemistry of the Main-Group Elements and their role in the Daily Life; y Neurociencia empresarial. Siete claves para la PYME.

4. Desarrollo de la plataforma. En el mes de mayo de 2015 se comenzó el desarrollo e implementación de la plataforma URJCx www.urjcx.es poniéndose en producción en el mes de noviembre de 2015.

5. Elaboración de los cursos. De forma paralela, desde el mes de junio de 2015, se comenzó el desarrollo de los cursos, con las siguientes subfases: a) Elaboración de las propuestas de contenidos (por parte de los expertos-profesores), b) Creación de diseño instruccional y guionización de los contenidos; c) Desglose de producción gráfica, audiovisual y multimedia (por parte de la Unidad de Producción de Contenidos Académicos de URJC online); d) Desarrollo gráfico y audiovisual (UPCA); e) Composición e integración multimedia en la plataforma (Unidad de Apoyo a la Docencia Virtual) y f) Testeo.

6. Despliegue de los primeros cursos. En el mes de enero de 2016 se comenzaron a impartir los primeros SPOCs (Sostenibilidad y Mejora de la Empleabilidad), y en el mes de febrero los primeros MOOC.

7. Evaluación. Una vez realizadas las primeras ediciones de los cinco cursos iniciales, se inició un proceso de evaluación integral del modelo, con el fin de analizar los resultados académicos, el emplazamiento de los cursos, el modelo de producción y los modelos de retorno. 


\section{Criterios de calidad en el diseño de los MOOC}

Para definir los criterios de calidad de los MOOC, se han analizado buena parte de las iniciativas que, sobre este particular, se han publicado en los últimos años tanto a través de modelos de estandarización sobre calidad en eLearning de forma general -UNE 66181:2012-, como específicamente sobre la calidad de los MOOC ADECUR- (Fernández, Silvera y Meneses, 2015).

De este modo, el diseño de los cursos recoge un conjunto de dimensiones pedagógicas que garanticen el aseguramiento de la calidad de los MOOC (Vila, Andrés y Guerrero, 2014) organizadas de forma específica en los siguientes ámbitos: a) Diseño metodológico: guía didáctica, metodología empleada y diseño instructivo motivador y adaptado a los destinatarios del curso; y b) Diseño de contenidos y de los recursos didácticos empleados: arquitectura de los contenidos, calidad de los contenidos (textuales, audiovisuales y multimedia), y diseño y calidad de las actividades de aprendizaje.

Además, tienen en consideración las características de un buen aprendizaje definidas por Conole (2013) y que se sustentan en fomentar principios como que los cursos inciten a la reflexión; estimulen y faciliten el diálogo mediante la creación de comunidades de aprendizaje; promuevan la colaboración; permitan aplicar los conceptos teóricos mediante el diseño de prácticas; estimulen la creatividad generando preguntas y abriendo la curiosidad por responderlas; y motiven al alumnado en ese proceso.

Considerando la clasificación de Conole (2013), el diseño de los cursos responde a un grado de apertura, masividad, densidad de comunicación y grado de colaboración media y un uso del multimedia, acreditación, formalidad, autonomía y diversidad alto.

\section{Metodología de desarrollo}

\section{Planteamiento general de los MOOC}

Los MOOC producidos bajo el modelo URJCx buscan motivar al alumnado abordando temáticas de interés masivo, orientadas sobre conocimientos previos pero sin pre-requisitos y centradas en aspectos sustantivos, precisos e impactantes seleccionados de entre la diversa oferta académica de la universidad.

Se adaptan a la planificación y a los ritmos personales del aprendizaje con una estimación real del esfuerzo del estudiante realizada, teniendo en cuenta situaciones o contextos de uso adverso y apoyada en una estructura flexible en su arquitectura de contenidos pero consistente y coherente en su experiencia de usuario. $Y$ se abren a la sociedad con su carácter claramente modular (Gértrudix et al., 2007), tanto en un diseño instructivo que se ajusta a la arquitectura de objetos educativos de jerarquía 
creciente -explicada por las administraciones educativas españolas en su "Guía para la aplicación del perfil de aplicación LOM-ES V1.o en la educación” (2009)- como en un despliegue de contenidos de distintos niveles (desde la secuencia de aprendizaje al media integrado) a través de las plataformas de gestión del aprendizaje y de los canales de difusión y redes sociales de la universidad.

Con estos principios, y con los datos y experiencia registrados en los primeros MOOC puestos a disposición de la comunidad educativa en los primeros meses de 2016, se ha configurado un estándar interno de calidad que se resume en los siguientes puntos de carácter orientativo:

- Esfuerzo estimado real del estudiante (consumo iterativo y comprensión del contenido, realización de actividades, estudio y evaluación) en torno a las 30 horas.

- Organización del curso (nivel de secuencia de aprendizaje) en cinco temas (nivel objeto de aprendizaje), con un despliegue inicial de todos los contenidos y con una dinamización y evaluación planteada en seis semanas: una por cada tema y otra complementaria para garantizar el ajuste a la disponibilidad real del alumnado, para llegar a una estimación real de unas 5 horas semanales de carga de trabajo del estudiante.

- El elemento multimedia fundamental es el vídeo que, a través de distintas tipologías (videoclase, videoapuntes, motion graphics, videos de animación, formatos televisivos, entrevistas, ficcionales...), se complementa con actividades de comprensión, con contenidos textuales e infográficos y con actividades de evaluación para constituir los objetos de aprendizaje que componen el MOOC.

Se trata de un modelo que pretende evolucionar desde lo que algunos autores definen como xMOOC típico:

One typical xMOOC consists of weekly structured learning contents. Within each week at least one video about the learning topic is provided as well as an additional content like weblinks, documents, or interactive learning objects. Finally, a weekly self-assessment should help students to foster their learning outcomes (Fischer et al., 2014, p. 5).

Estas orientaciones y parámetros de calidad se concretan en un diseño de producto que determina la metodología y la naturaleza de la producción y del desarrollo del MOOC. El modelo se sustenta en tres estructuras: la de los contenidos audiovisuales y multimedia; la del diseño instructivo (con el planteamiento de estrategias didácticas, la orientación docente, la dinamización de comunidades de aprendizaje o la evaluación) y la de la operación del curso (con su oferta de material adicional, la certificación y el seguimiento y evaluación del rendimiento de los usuarios del entorno virtual de aprendizaje). 
Figura 1. Modelo MOOC URJCx

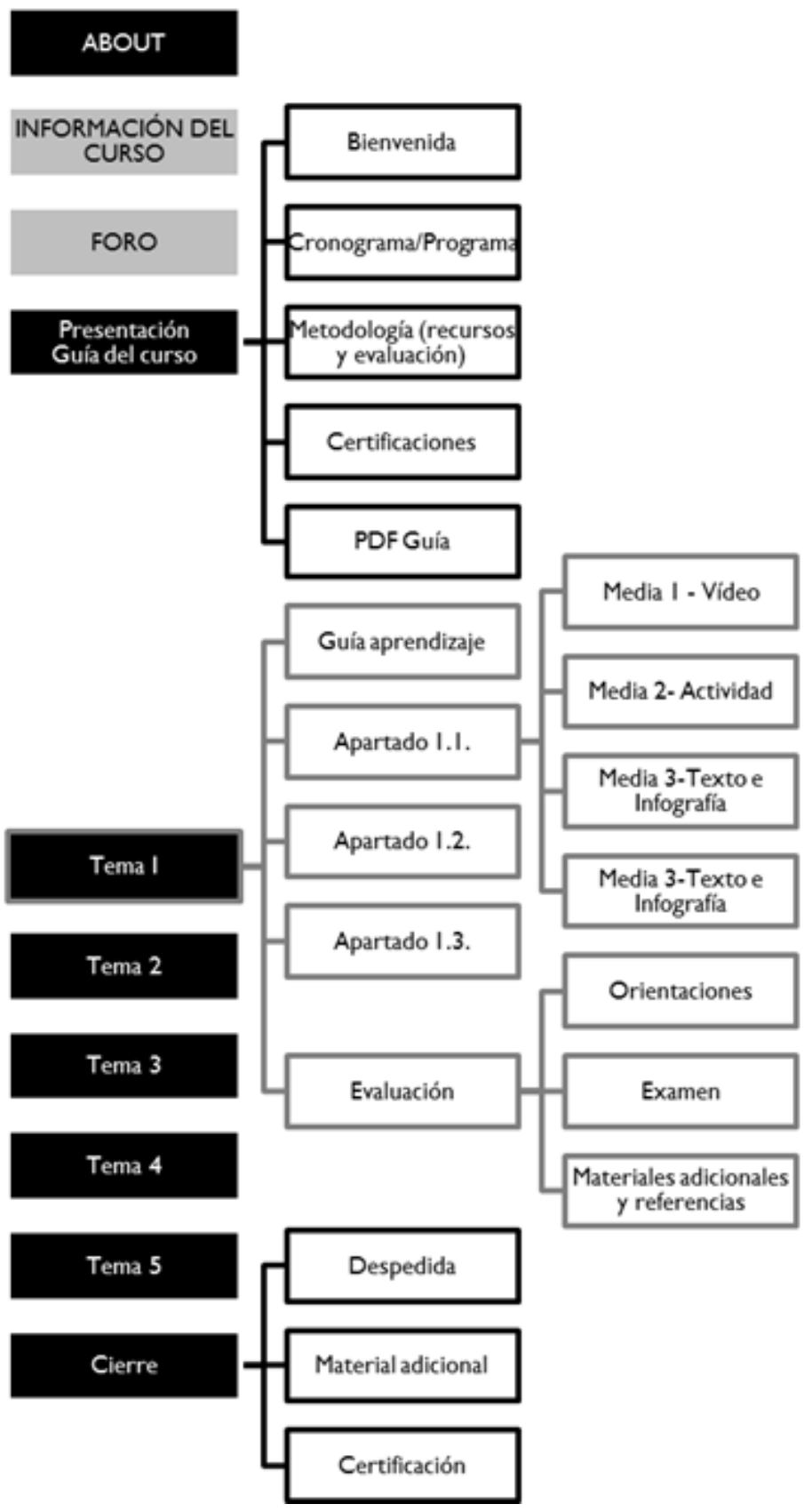




\section{Proceso de producción}

Dirigido por este diseño de producto, plasmado en un modelo de calidad que atiende a las estructuras de contenido, instructiva y de operación mencionadas, el proceso de producción de un MOOC URJCx se organiza en un ciclo que se sintetiza en la siguiente figura:

Figura 2. Ciclo de producción de un MOOC URJCx

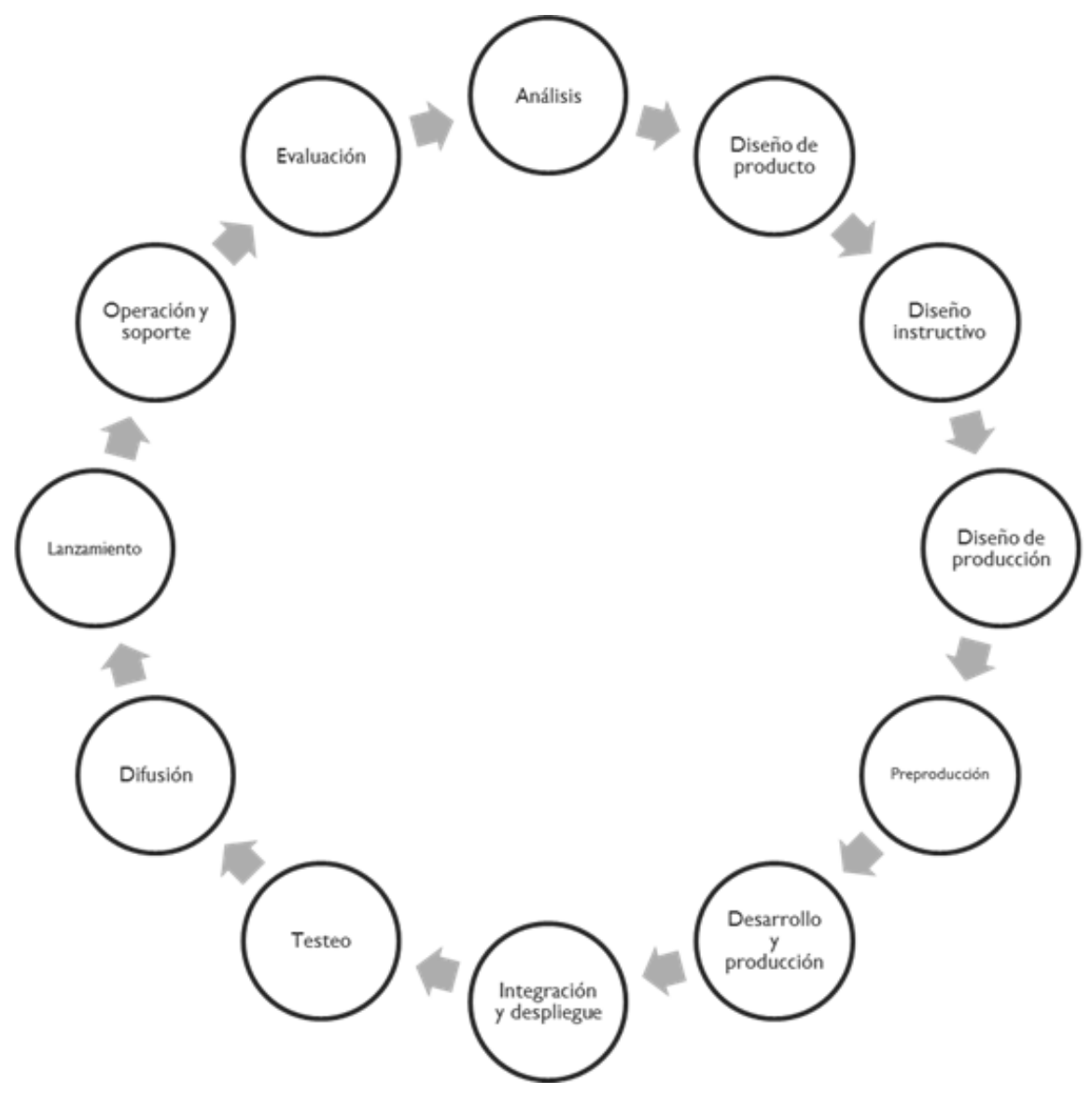

Se trata de un proceso en constante evolución, basado en un modelo metodológico híbrido de desarrollo, que es fruto de la adaptación a los recursos humanos y económicos disponibles y al contexto de producción en el que se desenvuelve. 
1. Análisis. Las tareas de análisis constituyen la pieza clave del desarrollo de los MOOC URJCx. Aunque teóricamente juegan el papel de punto de partida del proceso y puedan parecer asociadas al trabajo en cascada, en la práctica está presente en todos los momentos del diseño, de la producción y del seguimiento: las tareas, funciones, resultados y usos generan información valiosa sobre un fenómeno en constante evolución y al que hay que responder con rapidez y eficacia.

2. Diseño de producto. Orientado desde modelos cercanos al desarrollo de videojuegos, como la programación exploratoria, parte de una estructura inicial definida desde la investigación y el análisis de tendencias en el ámbito de los MOOC, pero va modificando sus especificaciones con la observación de las experiencias de producción y de uso. Genera una dinámica de reformulación constante de la serie o lote de cursos, aunque el contexto de producción aconseja la implementación de las evoluciones y mejoras en las producciones que se encuentren en los primeros momentos de desarrollo.

3. Diseño instructivo. Se realiza por docentes expertos en el área de conocimiento del curso pertenecientes a la universidad promotora de la acción, quienes la desempeñan de manera complementaria a sus obligaciones y funciones habituales. Es por ello una de las fases más determinadas por las restricciones de calendario y de disponibilidad de recursos, lo que obliga a configurarla con mayor rigidez en los plazos y en la linealidad de sus tareas.

4. Diseño de producción. Estrechamente ligado al diseño de producto, comparte su naturaleza dinámica y supera los obstáculos de los ajustes obligados de calendario y recursos con una alta capacidad para la identificación de errores en las experiencias de producción de los distintos MOOC. La planificación detallada y la normalización de procesos conviven de forma óptima con la flexibilidad necesaria para la prevención de problemas y desajustes en costes y uso de recursos.

5. Preproducción. Una de las etapas con mayor grado de evolución, del inicial trabajo en cascada a presentes métodos más iterativos y con la implicación y colaboración de los equipos docentes y de producción audiovisual y multimedia. Los desgloses de contenido y los guiones audiovisuales y multimedia ganan así en agilidad; la planificación compleja y detallada deja paso a la retroalimentación directa en reuniones de trabajo en las que las necesidades de producción se unen con los primeros pasos en el desarrollo de contenidos audiovisuales y multimedia.

6. Desarrollo y producción. Sujeta a un calendario y a una disponibilidad de recursos rígidos, obtiene el margen suficiente de flexibilidad gracias a la posibilidad de combinar tareas y compartir recursos y equipos con etapas habitualmente organizadas en momentos posteriores.

7. Integración, despliegue y testeo. En esta fase, las tareas de preproducción, producción, desarrollo y testeo se desempeñan con naturalidad y flexibilidad en el propio entorno virtual del MOOC, de manera que el producto final se va 
construyendo y cerrando de forma no lineal, guiado fundamentalmente por la optimización en la organización de equipos y recursos.

8. Difusión. Planificada inicialmente para coincidir con las últimas fases de desarrollo, previas al lanzamiento del curso, actualmente estas acciones responden a unos objetivos planteados con una mayor diversidad y a unas necesidades específicas de comunicación que determinan un calendario más amplio y que exigen una mayor continuidad y capacidad de reacción.

9. Lanzamiento, operación y soporte. En estas fases el MOOC cobra todo su sentido, está en manos de quien justifica su existencia, el alumnado. Son momentos cruciales, en los que el producto se expone al riesgo del fracaso, o lo que es lo mismo, a la oportunidad del éxito. La transparencia, la flexibilidad y la atención constante y personalizada al usuario desde los equipos docentes y de soporte son fundamentales para construir la necesaria comunidad de aprendizaje en la que temporalmente se convierte el curso; para enriquecer y hacer significativo el contenido desplegado; y para orientar a los estudiantes en la consecución de los objetivos didácticos detallados en la oferta de conocimiento realizada por la institución.

10. Evaluación. Y como en todo ciclo, un final es un nuevo principio. En este caso, entendiendo URJCx como una serie de conocimientos abiertos, la evaluación de la información generada por los usuarios mediante su interacción con la plataforma, con la comunidad de aprendizaje y con el equipo de soporte, alimenta e inicia el análisis previo del próximo curso.

\section{Equipos y flujo de trabajo}

El contexto de producción y el diseño de producto de los MOOC URJCx condicionan un flujo de trabajo con áreas estrechamente relacionadas y con equipos necesariamente especializados, pero intensamente conectados, orientados hacia la colaboración y formados en el conocimiento del modelo y sus resultados. 
Figura 3. Flujo de trabajo para la producción de un MOOC URJCx

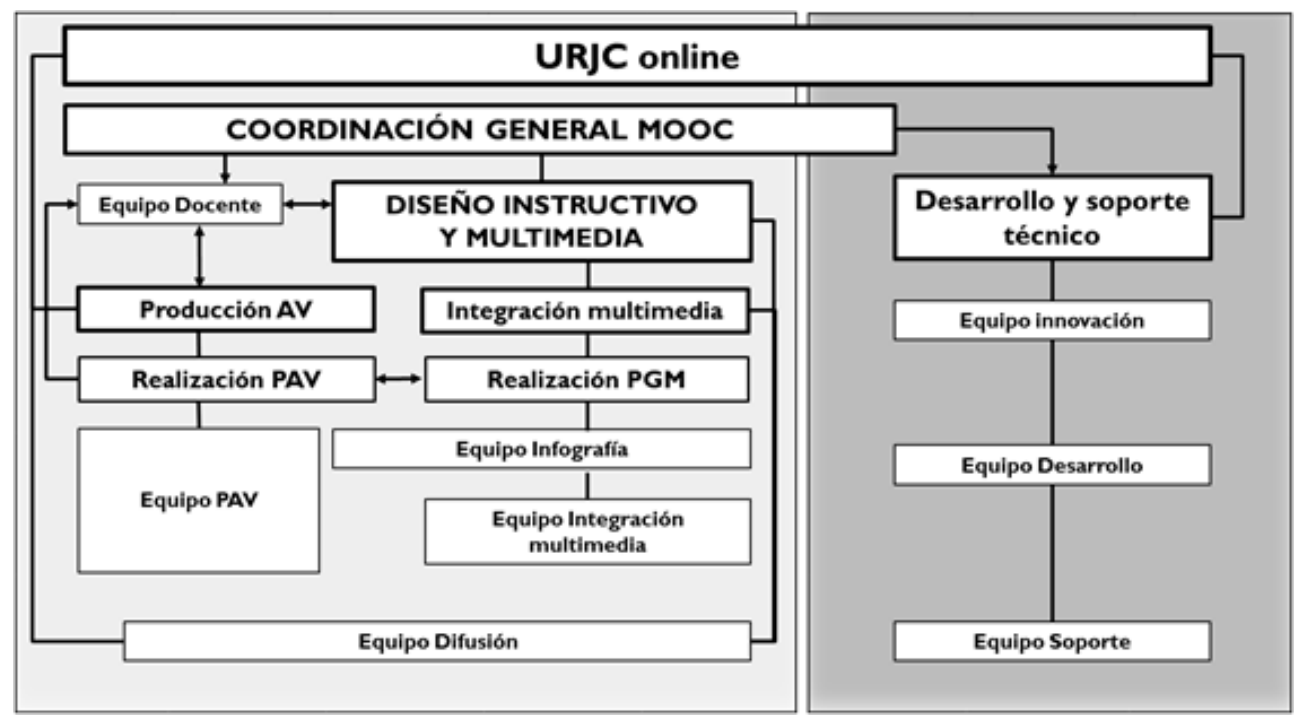

La dirección académica y la coordinación del Centro de Innovación en Educación Digital - URJC online constituyen el área dedicada a la configuración del contexto de producción de la serie y cada producto MOOC URJCx: gestionan y asignan recursos y determinan el calendario de lanzamiento y operación de los cursos.

La coordinación general del MOOC desempeña las funciones de diseño y gestión de producto. Se encarga, además, de proporcionar la formación necesaria a los distintos equipos implicados en la producción para que, más allá de su alta especialización en determinadas tareas y áreas, puedan trabajar en colaboración con el resto de equipos en las múltiples funciones conjuntas que entran en juego en el proceso de desarrollo. Y realiza el seguimiento y la evaluación de los resultados como instrumento imprescindible de mejora y evolución del producto y del servicio que se proporciona a la comunidad.

El área de diseño instructivo y multimedia comprende la configuración del producto en su dimensión de contenido digital educativo. En su seno se realiza el diseño instructivo y de la arquitectura de la información: selección de contenidos, estructura, elementos multimedia e interfaz de usuario. Se dirigen las acciones de producción multimedia, infográfica, interactiva y de integración y despliegue de contenidos; y se coordina al equipo docente en su aportación a la guionización multimedia y al flujo de comunicación y revisión del trabajo realizado por otros equipos y en el marco de otras áreas.

El área de soporte técnico desempeña funciones de investigación, desarrollo, innovación y mantenimiento de la plataforma que habilita el entorno virtual de 
aprendizaje del MOOC. Se organiza el trabajo relacionado con la gestión de usuarios (inscripción, matrícula y certificación); con el soporte técnico (atención al usuario mediante cuentas de correo, foros y otros canales de comunicación). Se proporciona asesoramiento a la coordinación general del curso en lo concerniente a soluciones de la plataforma y se recoge y filtra toda la información necesaria para el seguimiento, la evaluación y el análisis del modelo y de sus resultados y uso por el alumnado.

El área de producción audiovisual, con el vídeo como recurso característico y definitorio de los MOOC URJCx, merece un apartado específico.

\section{Producción audiovisual}

Algunos estudios empíricos revelan la importancia que posee el modelo de producción de vídeo en la capacidad de engagement que tiene en los estudiantes de MOOC. Algunos de los rasgos que hacen más efectivos los vídeos son: menor duración, cierta informalidad y vídeos con actividad como dibujar en una tablet (Guo, Kim y Rubin, 2014).

Por otra parte, las investigaciones de Hansch et al. (2015) en relación al valor instruccional central del vídeo en los MOOC, establecen algunos hallazgos sobre el coste de producción, la incierta relación entre el coste y la producción de valor en el aprendizaje, o las consecuencias de los procesos de estandarización de la producción de vídeo.

Por consiguiente, la producción de recursos audiovisuales para los MOOC debe fundamentarse, en primer lugar, en un análisis exhaustivo de las posibilidades de la comunicación videográfica o sonora aplicada a la elaboración de contenidos de naturaleza didáctica. Es decir, no se trata de realizar materiales audiovisuales que meramente reproduzcan o complementen contenidos de clases, apuntes u otros elementos textuales. El objetivo principal de la producción audiovisual debe añadir valor al proceso formativo para aprender más y mejor empleando medios diferentes a los tradicionales para ello. Traducir a imágenes y sonidos materiales que pueden transmitirse de una forma convencional no solo no aprovecha el potencial de las tecnologías audiovisuales aplicadas a la enseñanza online o en la difusión del conocimiento, sino que además, como resaltan los autores mencionados, se puede incurrir en disfuncionalidades evidentes en la relación coste-eficacia, ya que el esfuerzo de producción en términos económicos es muy alto, o alentar una estandarización de contenidos que, en lugar de aportar cualidades didácticas, dificultan o bloquean el proceso de enseñanza-aprendizaje. Por ejemplo, grabar una clase presencial convencional -una metodología de indudable y contrastada fuerza comunicativa- y convertirla en una pieza de vídeo lista para ser difundida por plataformas virtuales, no deja de desnaturalizar unos lenguajes, unos códigos didácticos que no estaban originalmente concebidos para ser transmitidos por ese canal tecnológico. Por ello, antes de replicar modelos de producción audiovisual hay 
que reflexionar sobre la naturaleza, modos, formatos, características y funciones de la creación videográfica para MOOC.

En el Centro de Innovación en Educación Digital esta fundamentación teóricopráctica sobre el papel medular que debe asumir la producción audiovisual en la enseñanza online o semipresencial es el primer eslabón de un proceso que no culmina con la creación de vídeos, podcasts o juegos interactivos, sino con la participación activa del espectador, del usuario, del alumno, cuando se difunde o se comparte una obra y este recurso visual o sonoro ha formado parte sustancial de su aprendizaje. Es en ese momento cuando puede medirse, evaluarse el impacto y la eficacia de dicho aprendizaje y la consecución de unos objetivos en cuanto a conocimientos, competencias o habilidades adquiridas.

Así, la apuesta por un modelo de producción determinado se establece a partir de tres vertientes: una tecnológica, que valora y selecciona los medios técnicos digitales más apropiados para la creación y distribución de contenidos; una narrativa y estética, que toma decisiones en el ámbito de la construcción de relatos audiovisuales originales, desde la escritura del guion a la postproducción, pasando por la dirección de actores, la fotografía o el diseño sonoro; $y$, sobre todo, una didáctica, que integra y guía la producción de materiales en función de su finalidad específicamente formativa.

En estos tres ámbitos se plantean las cuestiones fundamentales en la creación de contenidos para los MOOC: ¿Cómo integrar y difundir de forma natural materiales tan diversos en plataformas igualmente muy heterogéneas? ¿Qué infraestructuras o medios son necesarios para una producción de calidad y sostenible en el tiempo? ¿Cómo relacionar los contenidos audiovisuales entre sí y con otros materiales como documentación escrita, foros u otros recursos textuales? ¿Qué formatos audiovisuales incorporar? ¿Qué vinculación se establece entre el audiovisual didáctico con el cine, la televisión o la radio? ¿Cómo innovar en ámbitos como la interactividad o la inmersión del usuario en el relato? ¿Qué papel juegan los profesores y los alumnos en la creación de vídeos? ¿Cómo se consume, reedita y reformula el contenido realizado, partiendo de una filosofía libre de conocimiento abierto y de trabajo colaborativo? o ¿Cómo se evalúan los resultados de aprendizaje tras el visionado de un vídeo, por ejemplo?

Todos estos interrogantes se materializan en una serie de planteamientos básicos que vertebran la creación audiovisual llevada a cabo en la Unidad de Producción de Contenidos Académicos del CIED:

- El vídeo didáctico debe nutrirse de las técnicas y formatos de la realización cinematográfica, televisiva o radiofónica. En este sentido, ningún procedimiento, género o soporte está excluido de la producción audiovisual. Los formatos ficcionales (cortometrajes, series, etc.), informativos (reportajes, entrevistas, debates, etc.) o las tipologías propias del mundo académico (videoclases, 
videoapuntes, demostraciones con dispositivos, etc.) tienen cabida ya sea en vídeos de acción real o de animación, en sus múltiples manifestaciones creativas.

- Los MOOC son relatos de aprendizaje que se comunican a través de contenidos audiovisuales y multimedia, entre otros. Así, al entender la impartición o recepción de un curso como un viaje, una experiencia narrativa, se articulan los distintos materiales con el objetivo de enseñar y aprender aprovechando la potencialidad de la construcción audiovisual.

- La producción audiovisual deben llevarla a cabo tanto equipos cualificados (guionistas, editores, etc.) como docentes y alumnos, no solo en el diseño de los materiales curriculares sino en la propia realización: bien como actores, bien como técnicos, dentro de un proceso profesional de producción o como creadores independientes responsables de sus propios contenidos.

- En la creación de contenidos debe tenerse en cuenta la medición de la difusión y el impacto real, tanto a nivel cuantitativo como cualitativo, en el proceso de aprendizaje de la utilización -no unívoca o limitada a un canal concreto o un curso específico- de los vídeos en contextos mediáticos amplios como las redes sociales, las aulas u otros.

- Por último, la realización de vídeos o podcasts, entre otros, no es el único resultado al que deben dirigirse estos procesos tecnológicos: el diseño e implementación de nuevos dispositivos (como apps de grabación o postproducción, por ejemplo) es un campo que forma parte de la propia naturaleza informática de los MOOC dentro del ecosistema de Internet.

\section{Proceso}

La organización de la producción audiovisual estándar de un MOOC en URJCx, a partir de estas consideraciones, sigue un flujo de trabajo que consiste en las siguientes fases:

1. Gestión de fichas audiovisuales: contenido académico, formativo o didáctico del vídeo. Proceso de documentación del tema (conceptos, taxonomías, ejemplos, etc.) compilado por los docentes y que sirve de base a la preproducción.

2. Guionización audiovisual: conversión del tema a uno de los múltiples formatos audiovisuales. Escritura del vídeo e incorporación de técnicas de narrativa para enseñar y entretener al espectador-alumno.

3. Desglose y plan de grabación: necesidades de producción en cuanto a materiales gráficos, intérpretes, localizaciones, jornadas de grabación, etc.

4. Producción: siguiendo el cronograma, se captan las imágenes y sonidos o se crean las animaciones, ilustraciones o gráficos necesarios.

5. Postproducción: fase de edición y efectos visuales que da lugar a la finalización de la creación audiovisual. 
6. Catalogación y publicación: fundamental por la naturaleza abierta, flexible y colaborativa de los medios digitales. Fase del proceso que gestiona la difusión y utilización del material tanto como integrante de un curso como en su recuperación particular en el repositorio institucional de la universidad y/o en una plataforma abierta de distribución audiovisual en streaming como YouTube.

\section{CONCLUSIONES}

La puesta en marcha de una iniciativa para el diseño, desarrollo y puesta a disposición de $\mathrm{MOOC}$ requiere un fuerte apoyo institucional que se incardine dentro de los objetivos, de la misión y visión de la propia universidad. Exige estructuras de apoyo técnico pero también pedagógico, así como contar con recursos audiovisuales y multimedia suficientes. A nivel institucional representa tanto un reto como una oportunidad, y un motor de cambio en los modelos de despliegue de la oferta formativa.

El posicionamiento en favor de la potenciación del conocimiento abierto, mediante la aportación de contenidos y la gestión de cursos gratuitos y online, determina la configuración de un modelo de MOOC que se orienta hacia objetivos y características específicos cuyo valor ya se ha apuntado en párrafos anteriores: motivación, flexibilidad, modularidad y calidad.

La inversión material y humana que realiza la universidad para desplegar estos MOOC justifica la necesidad de trabajar para reducir las altas tasas de abandono que se producen, asumidas en muchos casos como parte inherente de su naturaleza de alcance masivo, pero fruto, en otros, de un modelo centrado en la lógica de la educación reglada y poco adaptado a las necesidades reales de un alumnado potencial de perfil heterogéneo, con demandas de aprendizaje específicas y con reducidas posibilidades de dedicación plena a cursos de este tipo.

El contexto de producción, determinado desde las primeras fases por la participación del profesorado de la institución promotora de los cursos -con una carga extra a sus tareas habituales-, incide notablemente en los métodos de desarrollo de los contenidos educativos digitales que se despliegan en estos MOOC. Esta metodología acaba caracterizándose por dos aspectos centrales: una alta dependencia del análisis y el seguimiento de los procesos y resultados de su aplicación; y una hibridación de enfoques y técnicas de producción que persiguen el ajuste a calendarios y presupuestos, evitar la repetición de errores y hacer evolucionar el modelo de desarrollo asociado al diseño de un producto educativo también en constante evolución para adaptarse a la demanda y al perfil de alumnado cambiantes.

El modelo de producción de vídeo coincide con el planteamiento de Hansch et al. (2015) de vídeos en segmentos cortos, separados por otros contenidos o actividades autoevaluativas. Se pone en discusión, no obstante, el valor añadido que ofrece el vídeo para el aprendizaje, considerando que si bien la calidad del audio es esencial, 
las producciones profesionales y de alto nivel, no siempre redundan en una mejora sustantiva del curso (Hansch et al. 2015). Aunque se coincide en que el diseño de producción debe ser consecuente con el contexto, la audiencia y la naturaleza del contenido del curso, los resultados obtenidos muestran que la óptima calidad de todo el producto, la diversidad de formatos y la granularidad de los procesos redunda en resultados globales más eficaces, aprovechables, reutilizables y con una vigencia mayor.

\section{REFERENCIAS BIBLIOGRÁFICAS}

Administraciones educativas españolas (2009). Anexo I de la Guía para la aplicación del Perfil de Aplicación LOMES V1.0 en la Educación. Educalab. Recuperado de http://educalab.es/intef/ tecnologia/recursos-digitales/lom-es/ guia

Aguaded, I. (2013). La revolución MOOCs, ¿una nueva educación desde el paradigma tecnológico? The MOOC Revolution: A New Form of Education from the Technological Paradigm? Comunicar, 41, 07-08. doi: http://dx.doi.org/10.3916/ C41-2013-a1

Aguaded, I., y Medina-Salguero, R. (2015). Criterios de calidad para la valoración y gestión de MOOC. RIED. Revista Iberoamericana de Educación a Distancia, 18(2), 119-143. doi: http:/ dx.doi.org/10.5944/ried.18.2.13579

Álvarez García, S. (2012). En busca del "Auleph". Aproximación a los entornos digitales para la gestión del aprendizaje. Revista ICONO14. Revista Científica de Comunicación y Tecnologías Emergentes, 8 (3), 303-327. doi: http:// dx.doi.org/10.7195/ri14.v8i3.241

Balkin, J. M., y Sonnevend, J. (2016). The Digital Transformation of Education. Education and Social Media: Toward a Digital Future. Cambridge, MA: MIT Press.

Bartolomé, A. R., y Steffens, K. (2015). ¿Son los MOOC una alternativa de aprendizaje? Are MOOCs Promising Learning Environments?. Comunicar, 44,
91-99. doi: http://dx.doi.org/10.3916 C44-2015-10

Best, S. (2015). Education in the interregnum: an evaluation of Zygmunt Bauman's liquid-turn writing on education. British Journal of Sociology of Education, 1-18.

Capdevilla Pagès, R., y Aranzadi Elejabeitia, P. (2014). Los Cursos Online Masivos y Abiertos: coportunidad o amenaza para las Universidades Iberoamericanas? RIED. Revista Iberoamericana de Educación a Distancia, 17(1), 69-82. doi: http://dx.doi.org/10.5944/ried.17.1.11574

Carr, W., y Kemmis S. (1988). Teoría crítica de la enseñanza. Barcelona: Martínez Roca.

Class-Central (2015). By The Numbers: MOOCS in 2015. Recuperado de https:/ www.class-central.com/report/moocs2015-stats

Conole, G. (2013). Los MOOCs como tecnologías disruptivas: estrategias para mejorar la experiencia de aprendizaje y la calidad de los MOOCs. Campus virtuales, 2(2), 16-28.

Contreras Espinosa, R., Eguia Gómez, J., y Solano Albajes, L. (2016). Investigaciónacción como metodología para el diseño de un serious game. RIED. Revista Iberoamericana de Educación a Distancia, 19(2). doi: http://dx.doi. org/10.5944/ried.19.2.15624

Elliott, J. (2005). El cambio educativo desde la investigación-acción. Madrid: Morata.

Fernández, M. B. R., Silvera, J. L. S., y Meneses, E. L. (2015). Comparativa entre 
instrumentos de evaluación de calidad de cursos MOOC: ADECUR vs Normas UNE 66181: 2012. Monográfico: Los MOOC: ¿una transformación radical o una moda pasajera?, 12(1), 131.

Fischer, H., Dreisiebner, S., Franken, O., Ebner, M., Kopp, M., y Köhler, T. (2014). Revenue vs. Costs of MOOC Platforms. Discussion of Business Models for XMOOC Providers Based on Empirical Findings and Experiences During Implementation of the Project iMOOX In 7th International Conference of Education, Research and Innovation (ICERI2014). IATED (pp. 2991-3000).

García Aretio, L. (2015). MOOC: ¿tsunami, revolución o moda pasajera? RIED: Revista Iberoamericana de Educación a Distancia, 18 (1), 9-21.

Gértrudix, M., Rajas, M., y Esteban, N. (2015). URJC online. Plan estratégico para la mejora de la enseñanza en entornos virtuales. In Formación virtual inclusiva $y$ de calidad para el siglo XXI: Actas del VI Congreso Internacional sobre Calidad y Accesibilidad de la Formación Virtual (CAFVIR 2015) (pp. 228-234).

Gértrudix, M., Álvarez, S., Galisteo, A., del Carmen Gálvez, M., y Gértrudix, F. (2007). Acciones de diseño y desarrollo de objetos educativos digitales: programas institucionales. RUSC. Universities and Knowledge Society Journal, 4(1).

Given, L. (2008). The SAGE Encyclopedia of Qualitative Research Methods. SAGE Publications.

González-Sancho, C., y Vincent-Lancrin, S. (2016). Transforming education by using a new generation of information systems. Policy Futures in Education. doi: 10.1177/1478210316649287

Guo, P. J., Kim, J., y Rubin, R. (2014). How video production affects student engagement: An empirical study of mooc videos. In Proceedings of the first ACM conference on Learning@ scale conference (pp. 41-50). ACM.
Hansch, A., Hillers, L., McConachie, K., Newman, C., Schildhauer, T., y Schmidt, P. (2015). Video and Online Learning: Critical Reflections and Findings from the Field.

Haywood, J., y Macleod, H. (2014). To MOOC or not to MOOC? University decision-making and agile governance for educational innovation. Massive Open Online Courses: The MOOC Revolution.

Hollands, F. M., y Tirthali, D. (2014a). Why Do Institutions Offer MOOCs?. Online Learning: Official Journal of the Online Learning Consortium, 18(3).

Hollands, F. M., y Tirthali, D. (2014b). MOOCs: Expectations and Reality. Full report. Online Submission.

INTEF (2016). ¿Qué es un NOOC? Recuperado de http://educalab.es/intef/ formacion/formacion-en-red/nood

Jarvis, J. (2010). Y Google, ¿cómo lo haría? Barcelona: Grupo Planeta (GBS).

López, E., Vázquez, E., y Román, P. (2015). Análisis e implicaciones del impacto del movimiento MOOC en la comunidad científica: JCR y Scopus (2010-13). Comunicar, 44, 73-80. doi: 10.3916/C442015-08

Peterson, R. (2013, 27 de septiembre). What do MOOCs cost? Minding the Campus. Recuperado de http://www. mindingthecampus.com/2013/og/what do moocs cost

Scopus (2010-13). Comunicar, 44, 73-80. doi: http://dx.doi.org/10.3916/C44 2015-08

Rifkin, J. (2014). La sociedad de coste marginal cero. Barcelona: Paidós.

Rodríguez, J. H. G., y González, P. A. V. (2013). Investigación Acción Participativa (IAP). Metodologías, Estrategias $y$ Herramientas Didácticas para el Diseño de Cursos en Ambientes Virtuales de Aprendizaje en la Universidad Nacional Abierta y a Distancia UNAD, 10.

Sánchez, M. M., León, M., y Davis, H. (2015). Desafíos en la creación, desarrollo e 
implementación de los MOOC: El curso de Web Science en la Universidad de Southampton. Challenges in the Creation, Development and Implementation of MOOCs: Web Science Course at the University of Southampton. Comunicar, 44.37-44. doi: http://dx.doi.org/10.3916/ C44-2015-04

Vila, R. R., Andrés, S. M., y Guerrero, C. S. (2014). Evaluación de la calidad pedagógica de los MOOC. Profesorado: Revista de curriculum y formación del profesorado, 18(1), 27-41.

White, T. J., Ran, L. H., Hou, R. N. J., Othman, B., y Ridwan, M. (2013). MOOCs: A diminution, evolution or revolution in University Teaching? In Educational Media (ICEM), 2013 IEEE 63rd Annual Conference International Council for. IEEE.

\section{PERFIL ACADÉMICO Y PROFESIONAL DE LOS AUTORES}

Manuel Gértrudix. Doctor en Ciencias de la Información por la UCM. Profesor de Comunicación Digital y Director Académico del Centro de Innovación en Educación Digital de la Universidad Rey Juan Carlos Universidad (España). Especialista en el área de Comunicación Digital, sus investigaciones se centran en el análisis de las posibilidades de las tecnologías emergentes aplicadas a diversos campos como la comunicación, las narrativas digitales o el eLearning. Ha sido Consejero Técnico de Nuevas Tecnologías del Ministerio de Educación y Ciencia de España. Es coordinador del grupo de investigación Ciberimaginario, co-editor de la revista científica ICONO14, y profesor del Master de Periodismo de Investigación, Datos y Visualización (URJC - Unidad Editorial), y del Máster de Analista de Inteligencia (URJC-UC3M). Ha sido investigador visitante en la Universidad Central de Florida (EE.UU.) y la Universidad de Stirling (Reino Unido).

E-mail: manuel.gertrudix@urjc.es

Mario Rajas-Fernández. Doctor en Ciencias de la Información por la UCM. Profesor de Lenguaje y Tecnologías Audiovisuales y Tecnologías Audiovisuales: Edición, en las titulaciones de Comunicación Audiovisual y Publicidad de la Universidad Rey Juan Carlos. Coordinador de la Unidad de Producción de Contenidos Académicos (UPCA) del Centro de Innovación en Educación Digital de la URJC. Es investigador del grupo de investigación Ciberimaginario, Editor de Icono14 Editorial y director de las colecciones 'Estudios de narrativa', 'Libros de arte' y 'Taller de experiencias audiovisuales'. Ha sido docente de la Facultad de Ciencias Sociales y Humanas de la Universidad Europea Miguel de Cervantes de Valladolid (20062008), y ha realizado estancias de investigación en el Tecnológico de Monterrey (México), la University of Aberystwyth (Gales) y la University of California Los Angeles (EEUU).

E-mail: mario.rajas@urjc.es

Sergio Álvarez-García. Profesor Asociado e investigador en la Universidad Rey Juan Carlos. Imparte formación de grado y postgrado en el campo de la comunicación 
multimedia. Ocupa el cargo de Coordinador de Proyectos de Contenidos en el Centro de Innovación en Educación Digital de la URJC. Acreditado como Contratado Doctor desde 2012, es doctor en Ciencias de la Información por la Universidad Complutense de Madrid. Sus líneas de investigación están relacionadas con la aplicación de las Tecnologías de la Información y la Comunicación al periodismo, la publicidad, la comunicación y el aprendizaje. Es investigador del grupo de investigación Ciberimaginario, secretario de la Asociación científica ICONO14, y profesor del Master de Periodismo de Investigación, Datos y Visualización (URJC - Unidad Editorial), y del Máster de Analista de Inteligencia (URJC-UC3M).

E-mail: sergio.alvarez@urjc.es

\section{DIRECCIÓN DE LOS AUTORES}

Facultad de Comunicación

Campus de Fuenlabrada

$\mathrm{C}^{\circ}$ del molino, $\mathrm{s} / \mathrm{n}$

28934 Fuenlabrada - Madrid (España)

Fecha de recepción del artículo: 01/06/2016

Fecha de aceptación del artículo: 30/07/2016

\section{Como citar este artículo:}

Gértrudix, M., Rajas-Fernández, M., y Álvarez-García, S. (2017). Metodología de producción para el desarrollo de contenidos audiovisuales y multimedia para MOOC. RIED. Revista Iberoamericana de Educación a Distancia, 2O(1), pp. 183203. doi: http://dx.doi.org/10.5944/ried.20.1.16691 